\title{
Avaliação do desempenho de diferentes métodos de estimativa da evaporação para duas regiões da Paraíba
}

\author{
Mário de M. V. B. R. Leitão ${ }^{1}$, Gertrudes M. de Oliveira ${ }^{2} \&$ Ted J. V. Leitão
}

\begin{abstract}
RESUMO
As regiões do Cariri e Sertão da Paraíba, nas cidades de Boqueirão e Patos, no período de 1 de abril de 2002 a 28 de fevereiro de 2003, foram estudadas com o objetivo de se avaliar o desempenho de diferentes métodos e obter-se as melhores estimativas da evaporação para essas regiões; para tanto, usaram-se medidas diárias de evaporação do tanque evaporimétrico padrão de $20 \mathrm{~m}^{2}$ (WMO, 1996). Os métodos avaliados foram: Penman (1948); balanço de energia - razão de Bowen; Linacre (1993); Snyder (1992); Kohler et al. (1955); Gangopadhyaya et al. (1966) e Hounam (1973). Os resultados indicaram que os métodos de estimativa de evaporação que utilizam a umidade relativa do ar, velocidade do vento e radiação solar, apresentaram os melhores desempenhos. O método proposto por Snyder (1992) teve o melhor desempenho nas duas regiões estudadas, com erros relativos de $-4,2 \%$ para Boqueirão e $-3,3 \%$ para Patos. O método de Linacre (1993), que utiliza os valores extremos de temperatura diária do ar, apesar de ter apresentado os maiores erros relativos $-16,9 \%$ em Boqueirão e -18,2\% em Patos, mostrou que, se for corretamente calibrado, pode ter desempenho satisfatório para as duas áreas estudadas.
\end{abstract}

Palavras-chave: radiação solar, umidade relativa, vento, recursos hídricos

\section{Evaluation of performance of different methods for estimation of evaporation in two areas of Paraíba}

\begin{abstract}
The municipalities of Boqueirão and Patos, during the period from April 1, 2002 to February 28, 2003, were studied to evaluate the different methods of obtainig evaporation. Daily measurements of evaporation of the standard tank of $20 \mathrm{~m}^{2}$ (WMO, 1996), and the Penman (1948); Linacre (1993); Snyder (1992); Kohler et al. (1955); Gangopadhyaya et al. (1966) and Hounam (1973) methods were used. The results indicated that the methods which use the relative humidity of air, wind speed and solar radiation presented the best performance. The method proposed by Snyder (1992) was the one that presented the best performance for the two studied areas, with the following relative error: $-4.2 \%$ for Boqueirão and $-3.3 \%$ for Patos. On the other hand, the method of Linacre (1993), which uses the daily air mean temperature obtained by the average of daily extreme values, presented the largest relative errors, $-16.9 \%$ in Boqueirão city and $-18.2 \%$ in Patos city, showing that, if calibrated correctly, it can provide satisfactory results for the two studied areas.
\end{abstract}

Key words: solar radiation, relative humidity, wind, water resources

1 UNIVASF, Av. Tancredo Neves 100, Centro, CEP 56306-410, Petrolina, PE. Fone: (87) 3862-2413. E-mail: mario.miranda@univasf.edu.br

2 UNEB-CAMPUS III, Av. Edgard Chastinet s/n, Bairro São Geraldo, CEP 48900-000, Juazeiro, BA. Fone: (74) 3611-7248. E-mail: gemoliveira@uneb.br 


\section{INTRODUÇÃO}

Muitas das equações empregadas no cálculo da taxa de evaporação são empíricas, o que as limita a serem usadas para locais e clima similar àquele onde foi efetuada sua determinação; desde modo e embora algumas dessas equações sejam freqüentemente citadas na literatura, é muito importante uma análise ao se usá-las, visto que elas podem não apresentar exatidão quando aplicadas para condições diferentes daquelas em que foram desenvolvidas (Oliveira, 2003). De acordo com Finch \& Gash (2002), medidas de taxas de evaporação ou variáveis meteorológicas básicas sobre superfícies aquáticas, são raramente disponíveis; em função disso, geralmente se adotam procedimentos para estimar a evaporação usando-se simples modelos baseados em medidas meteorológicas feitas sobre superfícies de solo seco; logo, o balanço de energia sobre corpos d'água pode ser diferente daquele sobre outras superfícies. Segundo Simon \& Mero (1985) devido a medida da evaporação real de superfícies de lagos não poder ser efetuada diretamente, é imprescindível estimála usando-se aproximações.

A estimativa da evaporação da água em açudes e barragens pode ser feita pela utilização de modelos fundamentados no balanço de energia, balanço de água, métodos aerodinâmicos e métodos combinados; neste sentido, é comum usar-se formulações empíricas ou semi-empíricas, não só para determinar os diferentes coeficientes para os modelos teóricos como, também, para obtenção indireta de determinados termos (parametrização) dos modelos evaporimétricos para lagos. Brutsaert \& Yeh (1970) comparando fórmulas empíricas baseadas no método de transferência de massa para estimar evaporação em lagos e tanques, concluíram que, embora essa teoria tenha limitações, representa uma ferramenta útil para o estudo da evaporação. Mero \& Simon (1975) ao efetuarem comparações entre alguns métodos de estimativa da evaporação, concluíram que o método do balanço de energia foi o que apresentou as melhores estimativas da evaporação para lago. Winter et al. (1995) afirmam que para uma base mensal a estimativa da evaporação de lago através da equação de Penman, produz um erro de cerca de $10 \%$ em comparação com o método do balanço de energia ou método do balanço de água. Chin \& Zhao (1995) afirmam que a evaporação é um dos principais componentes do ciclo hidrológico; logo, sua estimativa precisa é fundamental para o gerenciamento eficiente dos recursos hídricos.

Estudos que possibilitem a obtenção de medidas ou estimativas precisas de evaporação representam uma alternativa importante para o gerenciamento dos recursos hídricos, seja na atividade agrícola ou para armazenamento de água em reservatórios (Oliveira, 2003). Considerando que a otimização do uso dos recursos hídricos é um procedimento de suma significação para a região semi-árida, procurou-se avaliar o desempenho de alguns métodos de estimativa da evaporação, para as regiões do Cariri e Sertão da Paraíba, visando conhecer quais métodos possibilitam as melhores estimativas de evaporação.

\section{MATERIAL E MÉTODOS}

Os dados utilizados neste estudo foram coletados entre 01 de abril de 2002 a 28 de fevereiro de 2003, em duas áreas experimentais na Paraíba: uma na região do Cariri, na cidade de Boqueirão ( $07^{\circ} 29^{\prime} \mathrm{S}$; 36 $36^{\circ}$ 07’ $\mathrm{W}$; alt. $380 \mathrm{~m}$ ), situada próximo ao açude Epitácio Pessoa (Figura 1) e outra na região do Sertão, na cidade de Patos ( $07^{\circ} 01^{\prime}$ S; $37^{\circ} 17^{\prime}$ W; alt. $250 \mathrm{~m}$ ), localizada próximo ao açude Jatobá; as duas áreas experimentais estudadas estão separadas pelo Planalto da Borborema e distantes uma da outra, em cerca de $140 \mathrm{~km}$. Em cada área experimental foi instalado um tanque padrão de $20 \mathrm{~m}^{2}$ e $2 \mathrm{~m}$ de profundidade, recomendado pela Organização Meteorológica Mundial (WMO, 1996) para medida da evaporação em lagos, o qual foi impermeabilizado para evitar drenagem lateral e profunda. Medidas de evaporação de tanque Classe A, seguindo também padrões da WMO, foram efetuadas durante todo o período experimental.

Para determinação da evaporação diária foram efetuadas, diariamente, leituras, às $9 \mathrm{~h}$ nos citados tanques. Também foram medidas as seguintes variáveis climáticas: temperatura do ar e de bulbo úmido; temperatura da água dos tanques; velocidade do vento a 2 e $4 \mathrm{~m}$ acima do solo; direção do vento a $4 \mathrm{~m}$ acima do solo; radiação solar incidente; saldo de radiação a $1 \mathrm{~m}$ acima da superfície da água do tanque padrão e precipitação pluviométrica. Todos os sensores foram acoplados a um sistema automático de coleta de dados (micrologger 21X), o qual foi programado para efetuar leituras a cada segundo e médias a cada $30 \mathrm{~min}$.

Para estimar a evaporação foram utilizados os seguintes métodos:

O método de Penman (1948), recomendado pela FAO

$$
E_{L}=\left(s R n / L+\gamma E_{a}\right) /(s+\gamma)
$$

em que: Rn é o saldo de radiação sobre a água livre (cal $\mathrm{cm}^{-2} \mathrm{~d}^{-1}$ ); s é o coeficiente angular da curva de pressão de saturação $\left(\mathrm{hPa}{ }^{\circ} \mathrm{C}^{-1}\right)$; $\gamma$ é o parâmetro psicrométrico $\left(\mathrm{hPa}{ }^{\circ} \mathrm{C}^{-1}\right) ; \mathrm{E}_{\mathrm{a}}$ é o poder evaporante da superfície.

O coeficiente angular da curva de pressão de saturação (s) foi calculado pela expressão:

$$
\mathrm{s}=33,3639\left[0,05904\left(0,00738 \mathrm{~T}_{\mathrm{a}}+0,08072\right)^{7}-3,42 \times 10^{-5}\right]
$$

em que $\mathrm{T}_{\mathrm{a}}$ é a temperatura do ar, em ${ }^{\circ} \mathrm{C}$. Para obtenção de $\mathrm{E}_{\mathrm{a}}$ adotou-se:

$$
E_{a}=f(U)\left(e_{s}-e_{a}\right)
$$

em que: $\mathrm{U}$ é a velocidade do vento a $2 \mathrm{~m}$ de altura, em $\mathrm{km} \mathrm{d}^{-1}$; $\mathrm{e}_{\mathrm{s}}$ é a pressão de saturação (hPa); e é a pressão parcial de vapor (hPa). Conforme Doorenbos \& Pruitt (1977) f(U) é expresso por:

$$
f(U)=0,27(1+U / 100)
$$

O método do balanço de energia segundo a razão de Bowen simplificado para estimar evaporação em lagos: 


$$
E_{L}=\frac{R_{n}}{(1+\beta) L}
$$

em que: $\mathrm{R}_{\mathrm{n}}$ é a radiação líquida (cal $\left.\mathrm{cm}^{-2} \mathrm{~d}^{-1}\right)$; $\mathrm{L}$ é o calor latente de vaporização $(\mathrm{L}=595$ - 0,51t em cal g-1 , onde t é a temperatura do ar); e a razão de Bowen ( $\beta$ ) dada pela equação:

$$
\beta=\frac{\mathrm{c}_{\mathrm{p}} \mathrm{P}}{0,622 \mathrm{~L}}\left(\frac{\Delta \mathrm{T}}{\Delta \mathrm{e}}\right)
$$

em que: $c_{p}$ é o calor específico do ar $\left(c_{p}=0,24\right.$ cal g $\left.^{-1}{ }^{\circ} \mathrm{C}^{-1}\right)$; $\mathrm{P}$ é a pressão atmosférica (hPa); e $\Delta \mathrm{T}$ e $\Delta$ e são as diferenças de temperatura e pressão parcial do vapor d'água entre a superfície da água e $2 \mathrm{~m}$ acima da superfície do solo; $\gamma=\left(c_{p} \mathrm{P}\right) / 0,622 \mathrm{~L}$ é o parâmetro psicrométrico.

A equação para estimar a evaporação da água em lagos de Linacre (1993)

$E_{L}=\left(0,015+0,00042 T+10^{-6} z\right)\left[0,8 R_{s}-40+2,5 F u(T-T d)\right]$

em que: $E_{L}$ é a evaporação ( $\left.\mathrm{mm} \mathrm{d}^{-1}\right)$; $\mathrm{T}$ é a temperatura média diária do ar $\left({ }^{\circ} \mathrm{C}\right)$ obtida pela média dos valores extremos diários; z é a altitude do local (m); $\mathrm{R}_{\mathrm{s}}$ é a irradiância solar na superfície do lago $\left(\mathrm{W} \mathrm{m}^{-2}\right) ; \mathrm{F}=1,0$ - 8,7 x $10^{-5} \mathrm{z}$ é o fator de correção devido a altitude do local; u é a velocidade média do vento $\left(\mathrm{m} \mathrm{s}^{-1}\right)$ tomada a $2 \mathrm{~m}$ de altura da superfície e Td é a temperatura do ponto de orvalho $\left({ }^{\circ} \mathrm{C}\right)$.

O método do Tanque Classe A para estimar a evaporação em lagos, segundo Snyder

$$
\mathrm{E}_{\mathrm{L}}=\mathrm{K}_{\mathrm{p}} \mathrm{E}_{\mathrm{p}}
$$

em que: $E_{p}$ é a evaporação registrada no tanque classe $A$; $\mathrm{K}_{\mathrm{p}}$ o coeficiente de tanque geralmente assumido como sendo 0,66 e freqüentemente arredondado para 0,7, porém $K_{p}$ também pode ser estimado usando-se várias equações propostas como, por exemplo, a de Snyder (1992):

$$
\mathrm{K}_{\mathrm{p}}=0,482+0,024 \operatorname{Ln}(\mathrm{F})-0,000376 \mathrm{U}+0,0045 \mathrm{UR}
$$

em que: F é o tamanho da área de bordadura, em m; U é a velocidade do vento, em $\mathrm{km} \mathrm{d}^{-1}$; UR é a umidade relativa média do dia, em \%.

A equação de Penman (1948) modificada por Kohler et al. (1955)

$$
\mathrm{E}_{\mathrm{L}}=0,7\left(\frac{\mathrm{sR}}{\mathrm{s}+\gamma_{1}}+\frac{\gamma_{\mathrm{E}_{\mathrm{a}}}}{\mathrm{s}+\gamma_{1}}\right)
$$

em que $\gamma_{1}=0,000661 \mathrm{P}\left(\mathrm{kPa}^{\circ} \mathrm{C}^{-1}\right), \mathrm{E}_{\mathrm{a}}=\left(0,136+0,095 \mathrm{u}_{4}\right)$ $\left(\mathrm{e}_{\mathrm{s}}-\mathrm{e}_{2}\right)$ é o poder evaporante do ar $\left(\mathrm{mm} \mathrm{d}^{-1}\right), \mathrm{u}_{4}$ é a velocidade do vento a $4 \mathrm{~m}$ acima da superfície do solo $\left(\mathrm{m} \mathrm{s}^{-1}\right)$, $\mathrm{e}_{\mathrm{s}}$ é a pressão de saturação de vapor d'água a $2 \mathrm{~m}$ de altura (hPa), $\mathrm{e}_{2}$ é a pressão parcial do vapor a $2 \mathrm{~m}$ de altura $(\mathrm{hPa})$ e $\mathrm{R}_{\mathrm{n}}$ é o saldo de radiação, em equivalente lâmina de água evaporada $\left(\mathrm{mm} \mathrm{d}^{-1}\right)$.

O modelo de transferência de massa de Gangopadhyaya et al. (1966) \& Hounam (1973) que se baseia na equação de Dalton

$$
E_{L}=K\left(1+1,07 u_{2}\right)\left(e_{0}-e_{a}\right)
$$

em que, $\mathrm{E}_{\mathrm{L}}$ é dado em $\mathrm{mm} \mathrm{d}^{-1} ; \mathrm{u}_{2}$ é a velocidade do vento a $2 \mathrm{~m}$ de altura $\left(\mathrm{m} \mathrm{s}^{-1}\right)$; $\mathrm{e}_{\mathrm{o}}$ é a pressão de vapor na superfície da água (mb) e e é a pressão de vapor a $2 \mathrm{~m}$ (mb). O coeficiente empírico de ajuste $\mathrm{K}\left(\mathrm{mm} \mathrm{mb}^{-1} \mathrm{~d}^{-1}\right), \mathrm{K}$ foi tomado como 0,13 para o reservatório Epitácio Pessoa e como 0,14 para o reservatório Jatobá. Conforme Gangopadhyaya et al. (1966) e Hounam (1973), para grandes reservatórios K é igual a 0,13; para reservatórios de tamanho médio 0,14 e para pequenos reservatórios 0,15 .

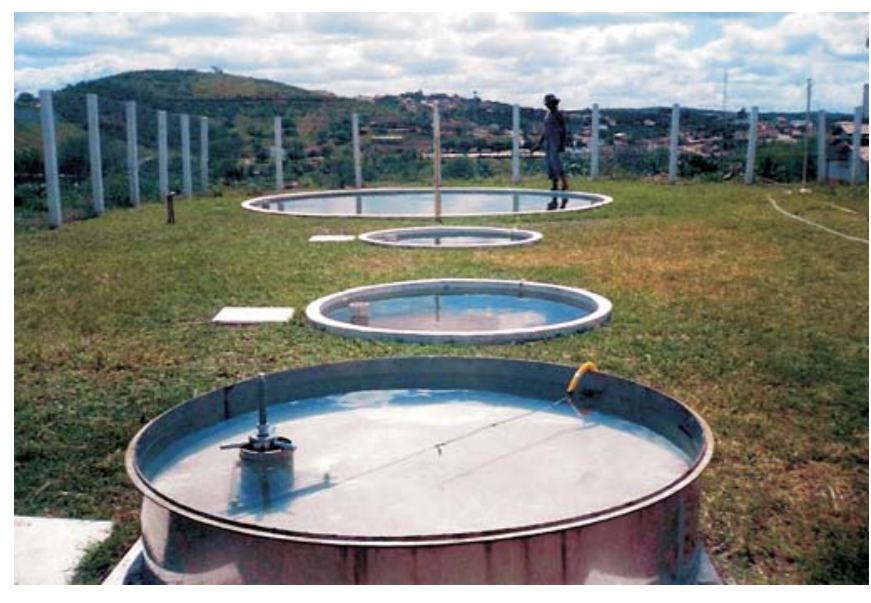

Figura 1. Tanques evaporimétricos na área experimental de Boqueirão

Para avaliar estatisticamente o desempenho dos métodos de estimativa de evaporação estudados, procurou-se correlacionar os valores estimados com as medidas, tomando-se como base os indicadores estatísticos propostos por Camargo \& Sentelhas (1997), definidos da seguinte forma: precisão - coeficiente de correlação "r"; exatidão - índice de Willmott "d” e de confiança ou desempenho "c" (Tabela 1).

A precisão é dada pelo coeficiente de correlação que indica o grau de dispersão dos dados obtidos em relação à média, ou seja, o erro aleatório. A exatidão está relacionada ao afastamento dos valores estimados em relação aos observados. Matematicamente, esta aproximação é dada por um índice designado concordância, representado pela letra "d" (Willmott et al., 1985), cujos valores variam de zero, para nenhuma concordância, a 1, para a concordância perfeita. O índice é dado pela seguinte expressão:

$$
\mathrm{d}=1-\left[\sum(\mathrm{Pi}-\mathrm{Oi})^{2} / \Sigma(|\mathrm{Pi}-\mathrm{O}|+|\mathrm{Oi}-\mathrm{O}|)^{2}\right]
$$

em que Pi é o valor estimado, Oi o valor observado e $\mathrm{O}$ a média dos valores observados.

O índice de confiança ou desempenho “c” para indicar o desempenho dos métodos segundo Camargo \& Sentelhas (1997), é representado pelo produto dos índices de precisão "r” e de exatidão “d”, expresso da seguinte forma:

$$
\mathrm{c}=\mathrm{r} * \mathrm{~d}
$$

Devido a estimativa de evaporação ser uma importante ferramenta para o planejamento adequado do uso da água e, conseqüentemente, para o monitoramente mais eficiente dos açudes no semi-árido do Nordeste brasileiro procurou-se 
Tabela 1. Critério de interpretação do desempenho dos métodos de estimativa da Evaporação, pelo índice " $c$ "

\begin{tabular}{cc}
\hline Valor de "c" & Desempenho \\
$>0,85$ & Ótimo \\
0,76 a 0,85 & Muito Bom \\
0,66 a 0,75 & Bom \\
0,61 a 0,65 & Mediano \\
0,51 a 0,60 & Sofrível \\
0,41 a 0,50 & Mau \\
$\leq 0,40$ & Péssimo \\
\hline
\end{tabular}

determinar, para todo o período estudado, os erros absoluto " $E_{\text {abs }}$ " e relativo " $E_{\text {rel }}$ " de cada método de estimativa em relação à evaporação medida. Para calcular os erros relativo e absoluto, usaram-se as expressões:

$$
\begin{gathered}
\mathrm{E}_{\mathrm{abs}}=\Sigma \mathrm{Pi}-\Sigma \mathrm{Oi} \\
\mathrm{E}_{\text {rel }}=[(\Sigma \mathrm{Pi}-\Sigma \mathrm{Oi}) / \Sigma \mathrm{Oi}] 100
\end{gathered}
$$

em que Pi é o valor estimado e Oi o valor observado.

\section{RESULTADOS E DISCUSSÃO}

Comparando-se as curvas de estimativa de evaporação dos diversos métodos com a curva da evaporação observada no tanque padrão de $20 \mathrm{~m}^{2}$ para Boqueirão durante o período estudado, apresentadas na Figuras 2, verifica-se que as estimativas pelo método de Penman (1948) apesar de sua aproximação com as medidas entre junho e o final de agosto, em abril e a partir de setembro até fevereiro, apresentaram subestimação. As estimativas pelo método do balanço de energia - BE, sobreestimaram as medidas ao longo de todo o período estudado. Verifica-se, ainda, que as estimativas pelo método de Gangopadhyaya et al. (1966) e Hounam (1973) - G\&H mostraram uma aproximação relativa com as medidas entre abril e julho e, a partir daí até fevereiro, uma sobreestimação, que foi mais acentuada entre setembro e dezembro; já as estimativas pelo método de Linacre (1993) mostraram uma tendência mais ou menos constante de subestimação das medidas, durante todo o período estudado. No caso das estimativas pelo método de Snyder (1992), apesar de terem subestimado as medidas entre outubro e janeiro foram, de modo geral, as estimativas que mais se aproximaram das medidas. Finalmente, as estimativas pelo método de Kohler et al. (1955), subestimaram as medidas de abril a maio, apresentaram aproximação de junho a agosto e as sobreestimaram de setembro a fevereiro, porém, fazendo-se uma análise do desempenho de cada método de estimativa de evaporação para Boqueirão, em termos da magnitude dos erros relativo e absoluto cometidos para todo o período estudado verifica-se, através da Tabela 2, que os menores erros relativo e absoluto em Boqueirão foram cometidos pelo método de Snyder (1992), e os maiores pelo método de Linacre (1993).

Comparando-se as curvas de estimativa de evaporação dos diversos métodos com a curva da evaporação observada no tanque padrão de $20 \mathrm{~m}^{2}$ para Patos durante o período estuda- do, apresentadas na Figuras 3, verifica-se que as estimativas obtidas pelo método de Penman indicaram uma aproximação relativa com as medidas no início do período, de abril a maio, bem como no final do período, de dezembro a fevereiro, porém no período intermediário, de maio a dezembro, mostraram subestimação. As estimativas pelo método do Balanço de Energia durante todo o período estudado tenderam a uma sobreestimação das medidas, enquanto as estimativas pelo método de Gangopadhyaya et al. (1966) e Hounam (1973) mostraram uma aproximação entre abril e outubro, subestimação de novembro a dezembro e sobreestimação de janeiro a fevereiro; já as estimativas pelo método de Linacre, exceto no final do período estudado, de janeiro a fevereiro, mostraram uma aproximação relativa com as medidas mas, no restante do período apresentaram uma subestimação praticamente constante e mais acentuada que em Boqueirão. Também em Patos as estimativas pelo método de Snyder (1992) mostraram, durante todo o período estudado, uma boa aproximação com as medidas. Finalmente, as estimativas pelo método de Kohler et al. (1955) apresentaram subestimação de abril a junho, aproximação com as medidas de julho a dezembro e uma sobreestimação de dezembro a fevereiro. Da mesma forma que em Boqueirão, e se fazendo uma análise para Patos do desempenho de cada método de estimativa de evaporação, com base na magnitude dos erros relativo e absoluto cometidos para todo o período estudado verifica-se, através da Tabela 2, que o método de Snyder (1992) apresentou os menores erros relativo e absoluto, enquanto o método de Linacre (1993) foi o que proporcionou os maiores erros.

\begin{tabular}{|c|c|c|c|c|c|c|}
\hline \multirow[b]{2}{*}{ Método } & \multicolumn{3}{|c|}{ Boqueirão } & \multicolumn{3}{|c|}{ Patos } \\
\hline & $\begin{array}{l}E_{\text {rel }} \\
(\%)\end{array}$ & $\begin{array}{l}E_{a b s} \\
(m m)\end{array}$ & $\begin{array}{l}\text { Magnitude } \\
\text { do erro }\end{array}$ & $\begin{array}{l}E_{\text {rel }} \\
(\%)\end{array}$ & $\begin{array}{l}E_{a b s} \\
(\mathrm{~mm})\end{array}$ & $\begin{array}{c}\text { Magnitude } \\
\text { do erro }\end{array}$ \\
\hline Snyder & 4,2 & 74,9 & menor & $-3,3$ & $-64,3$ & menor \\
\hline$G \& H$ & $-5,9$ & $-105,8$ & - & $-4,1$ & $-81,7$ & - \\
\hline Kohler & 9,1 & 164,3 & - & 5,0 & 99,4 & - \\
\hline Penman & $-9,8$ & $-177,1$ & - & $-10,7$ & $-210,5$ & - \\
\hline $\mathrm{BE}$ & 13,1 & 236,0 & - & 12,0 & 237,4 & - \\
\hline Linacre & $-16,9$ & $-303,8$ & maior & $-18,2$ & $-360,3$ & maior \\
\hline
\end{tabular}

Tabela 2. Valores dos erros relativos e absolutos de cada método

Apresenta-se, na Tabela 3 e Figuras 4 e 5, o desempenho dos métodos de estimativa de evaporação com base nos indicadores estatísticos de Camargo \& Sentelhas (1997), em que a precisão é dada pelo coeficiente de correlação “r”, a

Tabela 3. Desempenho dos métodos de estimativa da evaporação, segundo o índice de desempenho " $c$ ", em correlação com dados de tanque evaporimétrico padrão $20 \mathrm{~m}^{2}$ para Boqueirão e Patos

\begin{tabular}{lcccccc}
\hline \multirow{2}{*}{ Método } & \multicolumn{2}{c}{ Boqueirão } & & \multicolumn{2}{c}{ Patos } \\
\cline { 2 - 3 } \cline { 6 - 6 } Penman (1948) & 0,71 & Bom & & 0,54 & Sofrível \\
Balanço de Energia & 0,77 & Muito Bom & & 0,66 & Bom \\
Gangopadhyaya et al. & 0,74 & Bom & & 0,73 & Bom \\
(1966) e Hounam (1973) & 0,74 & & & & & \\
Linacre (1993) & 0,78 & Muito Bom & & 0,66 & Bom \\
Snyder (1992) & 0,91 & Ótimo & & 0,89 & Ótimo \\
Kohler et al. (1955) & 0,75 & Bom & & 0,60 & Sofrível \\
\hline
\end{tabular}


exatidão representada pelo índice de Willmott “d” e de confiança ou desempenho por “c”, para Boqueirão e Patos, respectivamente. Verifica-se, pela Tabela 3 que, de acordo com os indicadores citados, o método de Snyder (1992) apresen-

A.

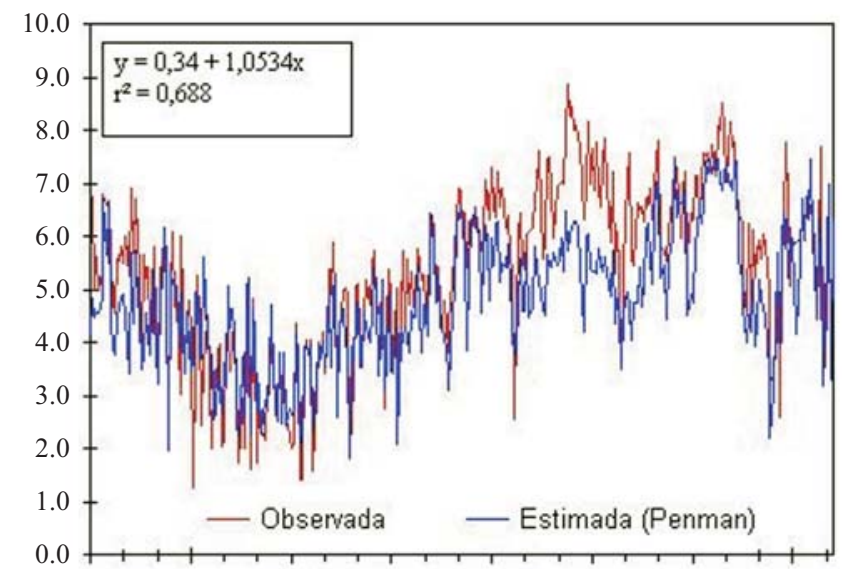

C.

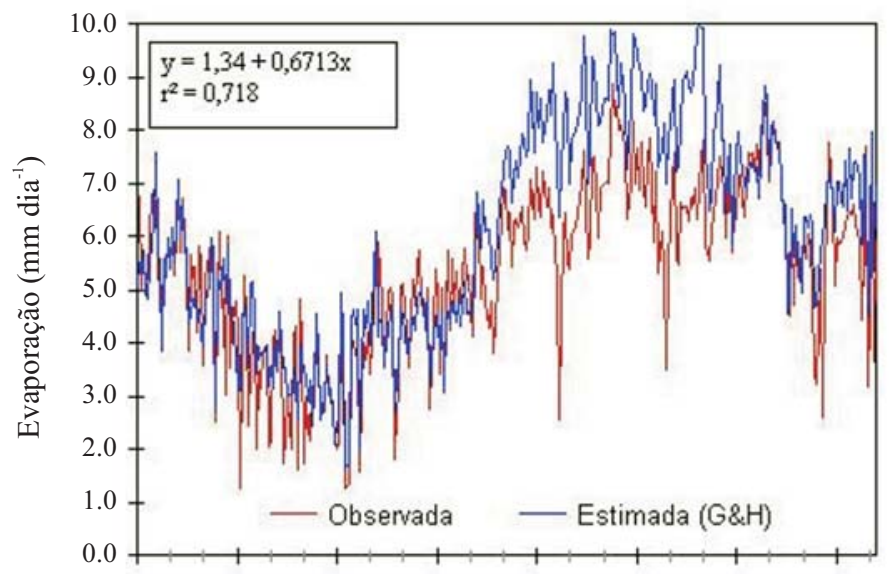

E.

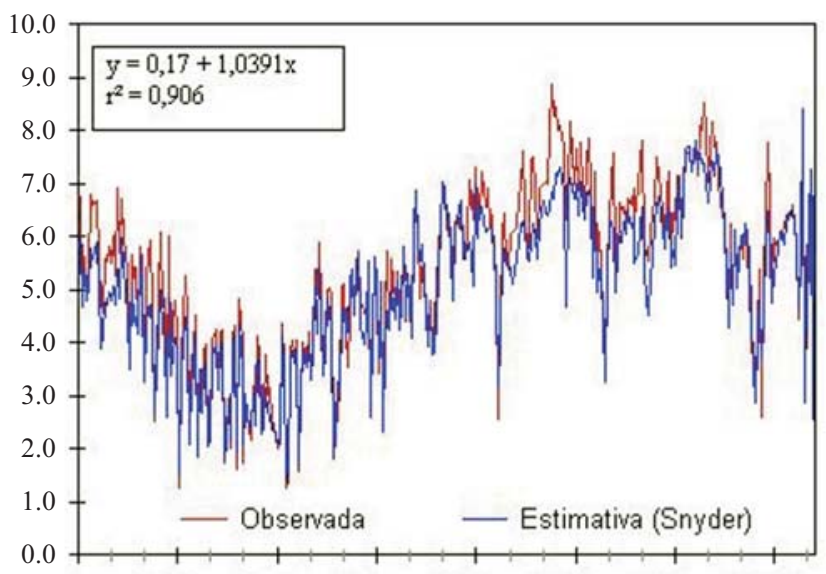

01-Apr 16-May 30-Jun 14-Aug 28-Sep 12-Nov 27-Dec 10-Feb tou o melhor desempenho sendo classificado, portanto, como ótimo, em Boqueirão e Patos; o método do Balanço de energia e o método de Linacre (1993) tiveram, respectivamente, desempenho muito bom em Boqueirão e bom em

B.

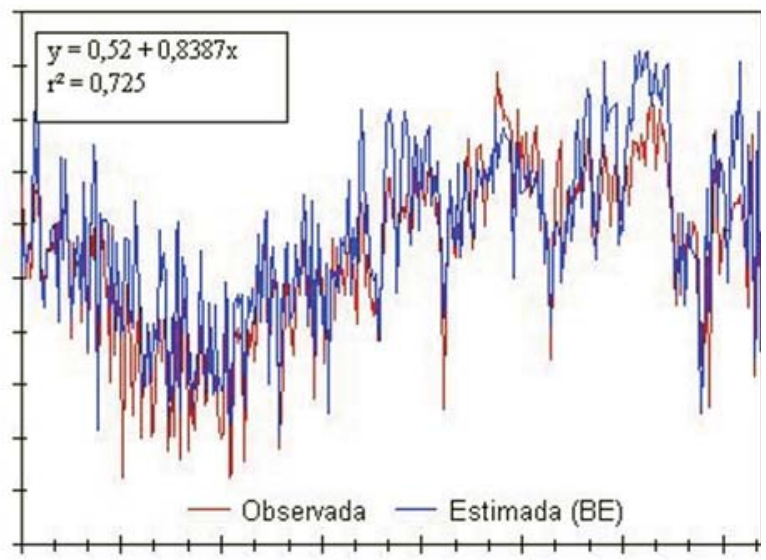

D.

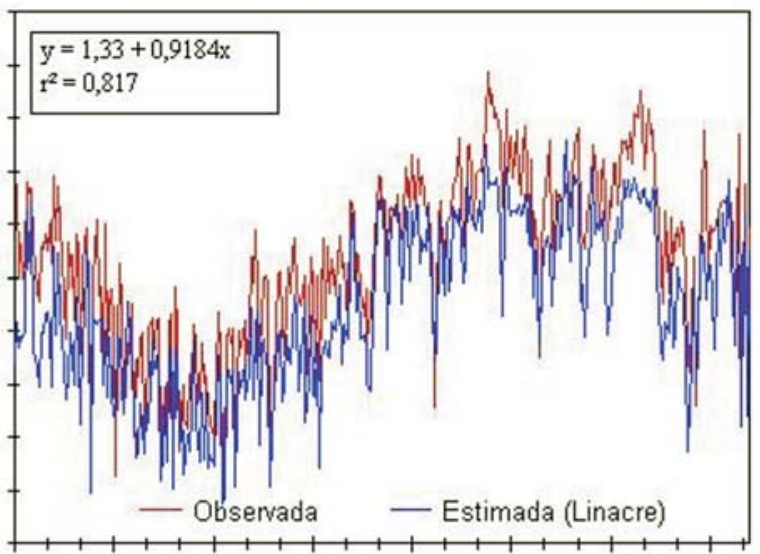

F.

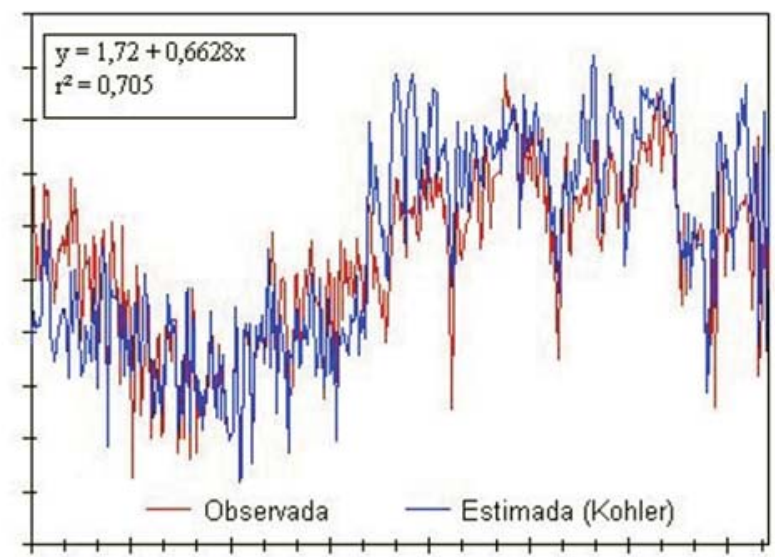

01-Apr 16-May 30-Jun 14-Aug 28-Sep 12-Nov 27-Dec 10-Feb

Período de observação

Figura 2. Comparação, para Boqueirão, da evaporação estimada pelos métodos de: (A) Penman (1948); (B) balanço de energia - BE; (C) Gangopadhyaya et al. (1966) e Hounam (1973) - G\&H; (D) Linacre (1993); (E) Snyder (1992) e (F) Kohler et al. (1955), em relaçã̃o à evaporação observada 
A.

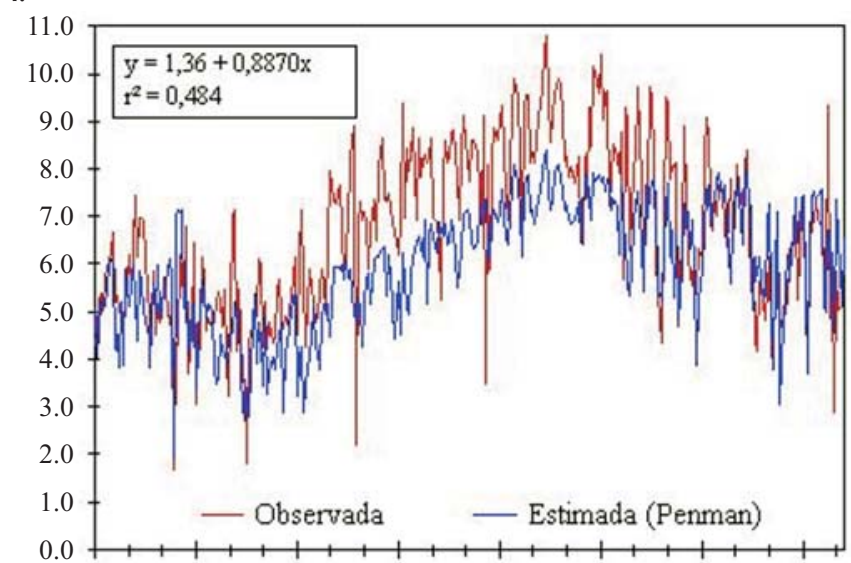

C.

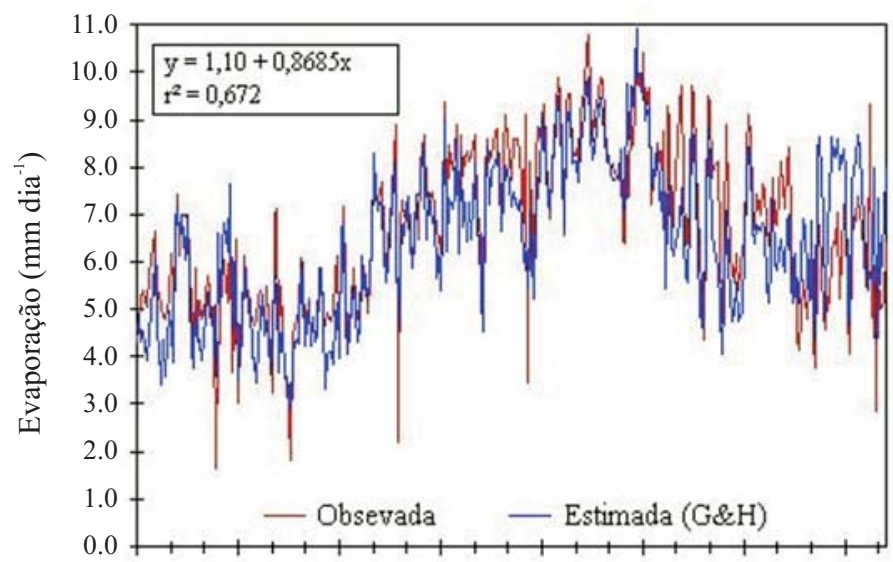

B.

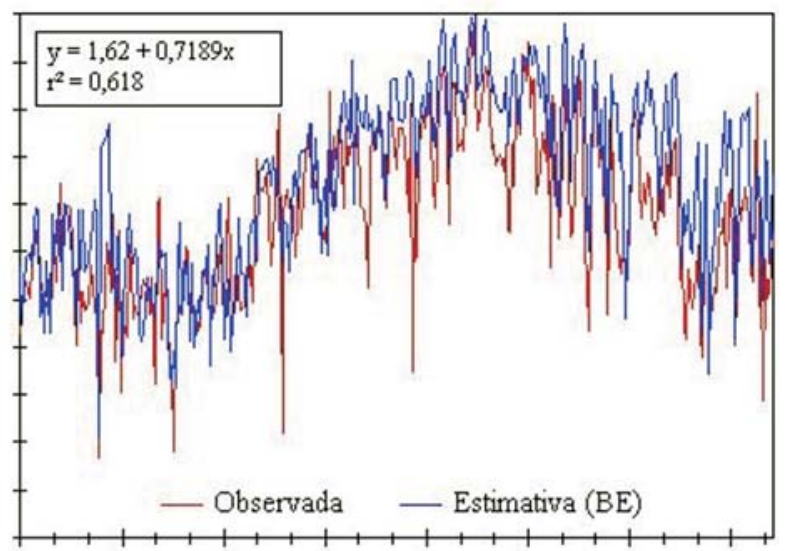

D.

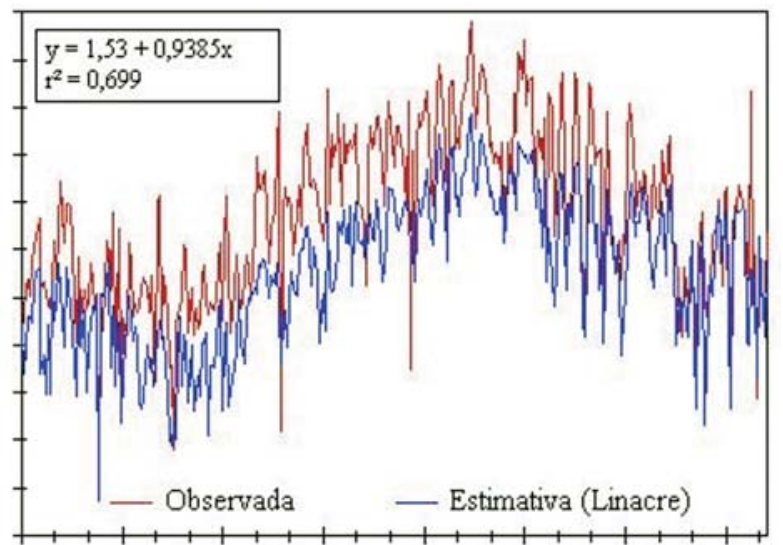

E.

F.

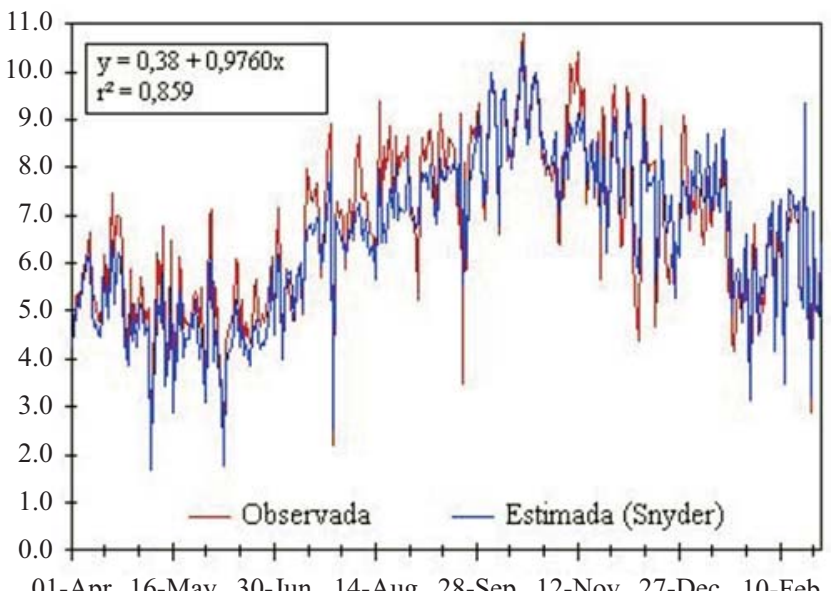

01-Apr 16-May 30-Jun 14-Aug 28-Sep 12-Nov 27-Dec 10-Feb

Período de observação

Figura 3. Comparação, para Patos, da evaporação estimada pelos métodos de: (A) Penman (1948); (B) balanço de energia - BE; (C) Gangopadhyaya et al. (1966) e Hounam (1973) - G\&H; (D) Linacre (1993); (E) Snyder (1992) e (F) Kohler et al. (1955), em relação à evaporação observada

Patos; o método de Gangopadhyaya et al. (1966) e Hounam (1973) o desempenho foi bom, tanto em Boqueirão como em Patos; os métodos de Penman (1948) e Kohler et al. (1955) mostraram desempenho bom em Boqueirão, porém ambos tiveram desempenho sofrível em Patos. Com base nos indica- dores estatísticos de Camargo \& Sentelhas (1997), todos os métodos de estimativa de evaporação usados neste trabalho tiveram desempenho satisfatório em Boqueirão; já em Patos apenas os métodos de Penman (1948) e Kohler et al. (1955) não apresentaram desempenho satisfatório. 
Em resumo, o método de Snyder (1992), que considera não só a velocidade do vento mas também a umidade relativa (UR), mostrou ótimo desempenho tanto no que diz respeito à análise qualitativa, com a aproximação da curva da evaporação estimada em relação à curva da evaporação observada, como em relação às análises quantitativas:

A.

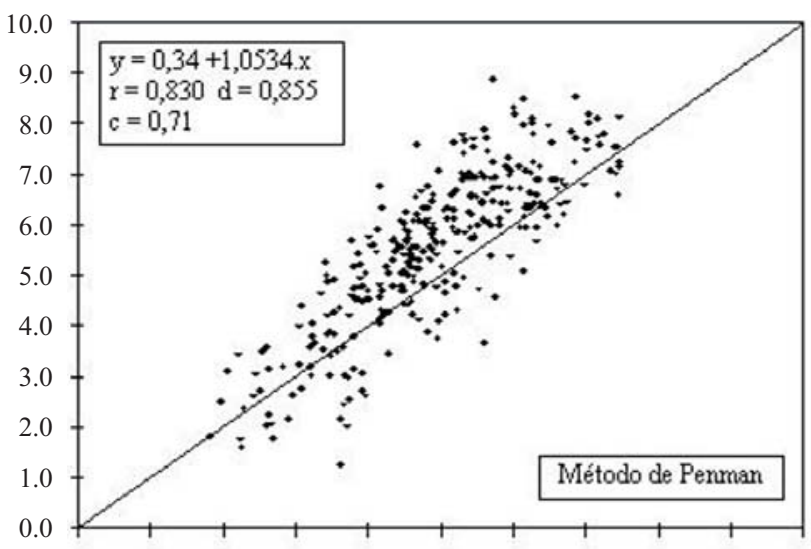

C.

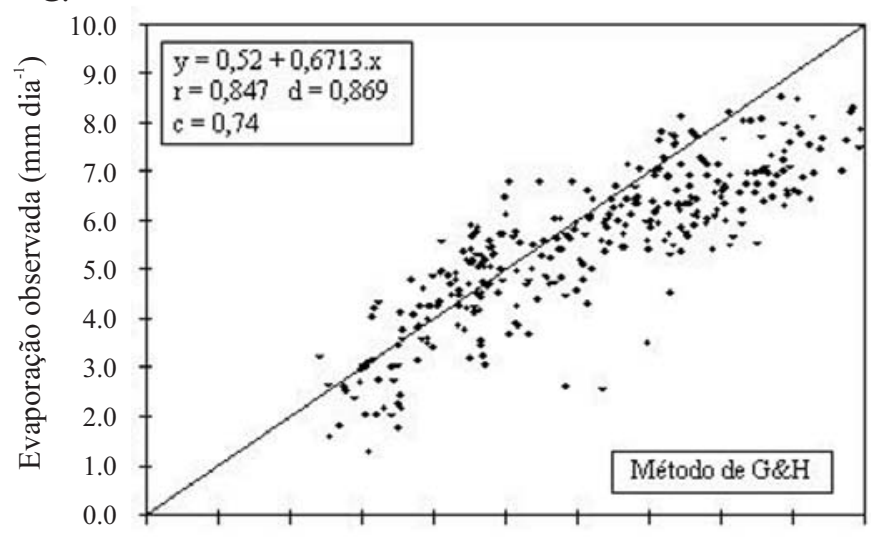

E.

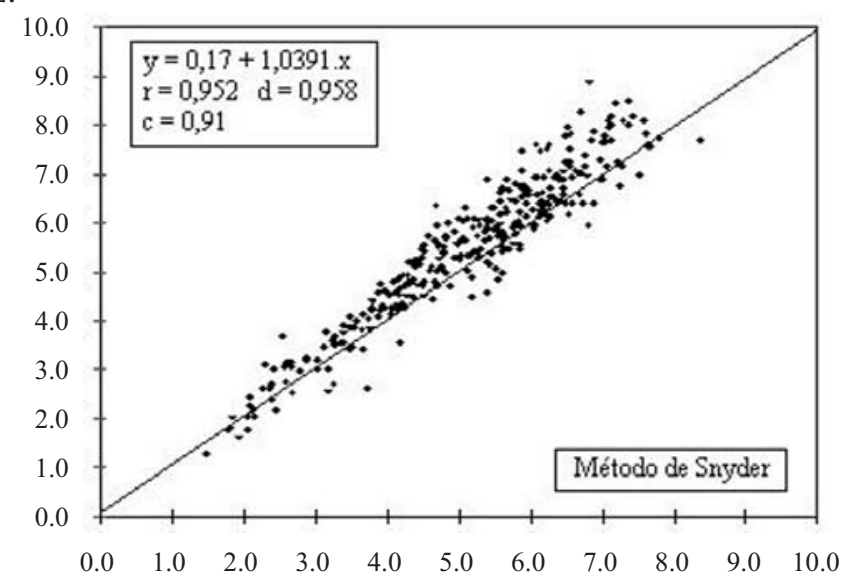

erros relativo e absoluto aceitáveis (conforme a Tabela 2 Boqueirão: $\mathrm{E}_{\mathrm{rel}}=-4,2 \%$ e $\mathrm{E}_{\mathrm{abs}}=-74,9 \mathrm{~mm}$; Patos: $\mathrm{E}_{\mathrm{rel}}=$ $-3,3 \%$ e $\left.\mathrm{E}_{\mathrm{abs}}=-64,3 \mathrm{~mm}\right)$; coeficiente de determinação " $\mathrm{r}^{2}$ " e levado (conforme indicado nas Figuras 2 e 3 - Boqueirão: 0,906; Patos: 0,859) e coeficiente de confiança "c" também elevado (conforme indicado nas Figuras 4 e 5 -

B.

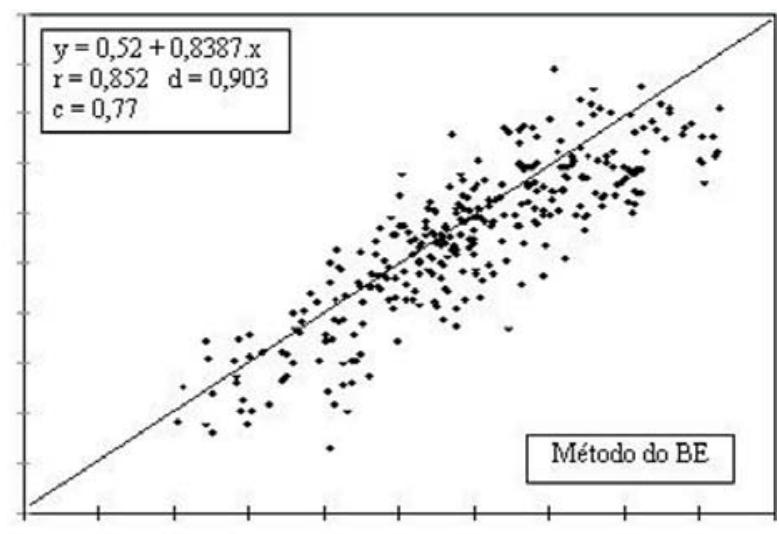

D.

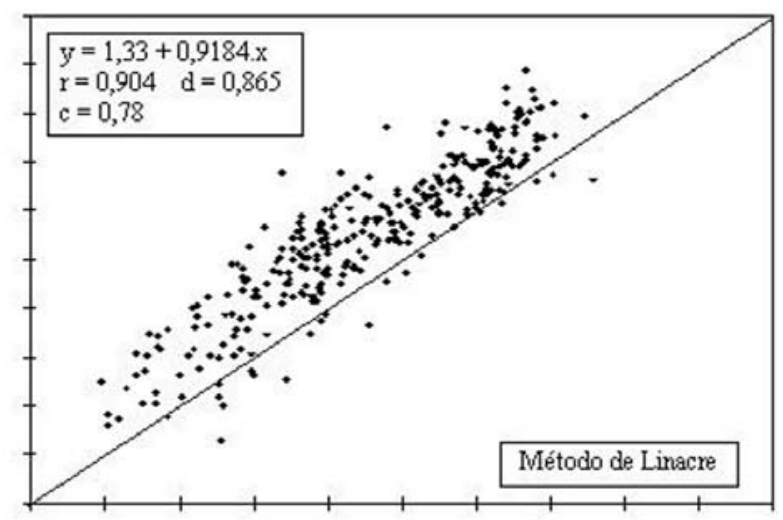

F.

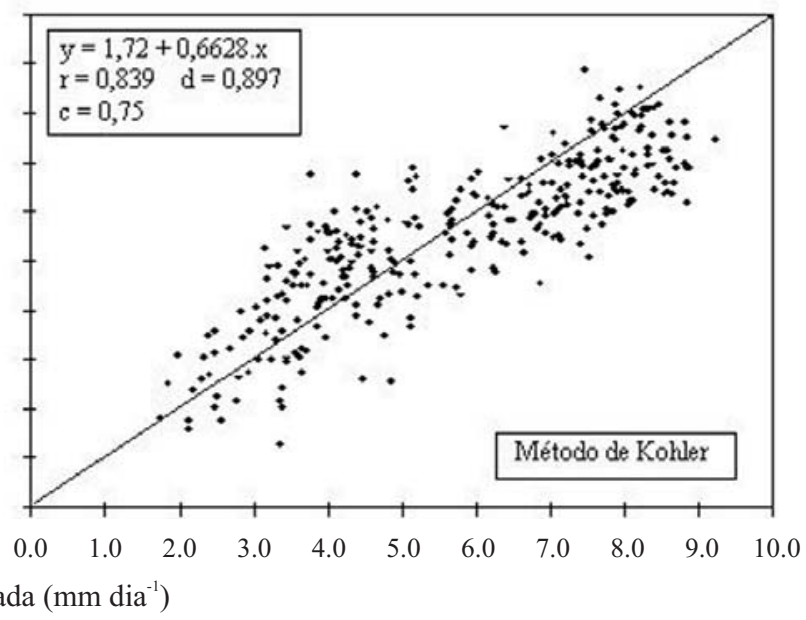

Figura 4. Dispersão para Boqueirão, $\mathrm{PB}$, da evaporação estimada pelos métodos de (A) Penman (1948); (B) balanço de energia - BE; (C) Gangopadhyaya et al. (1966) e Hounam (1973) - G\&H; (D) Linacre (1993);(E) Snyder (1992) e (F) Kohler et al. (1955), em relação à evaporação 
A.

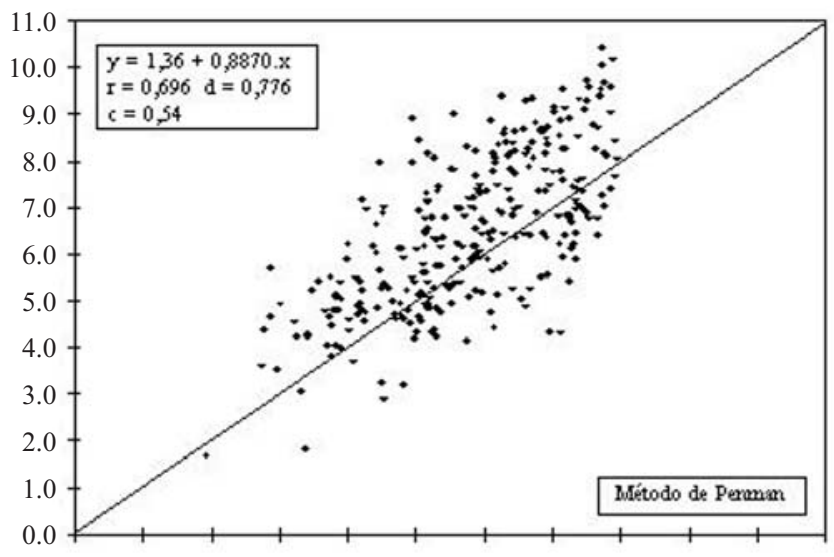

C.

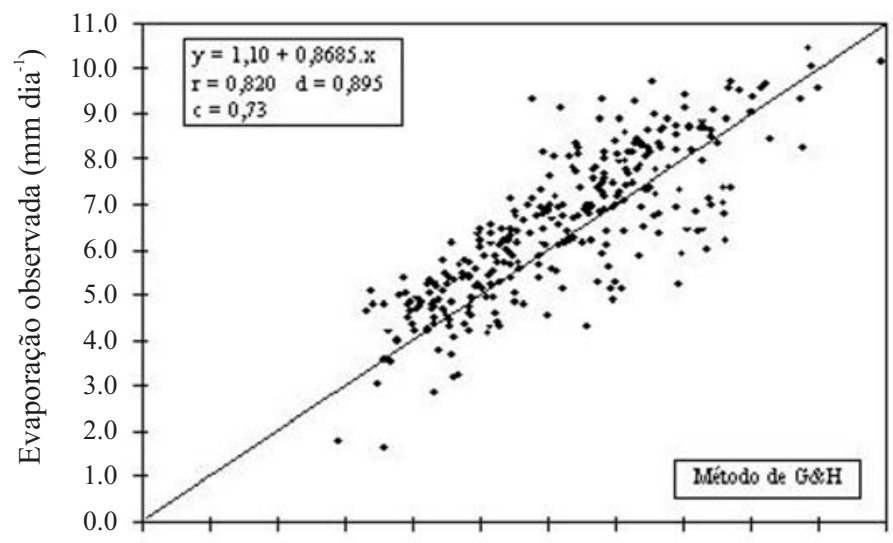

E.

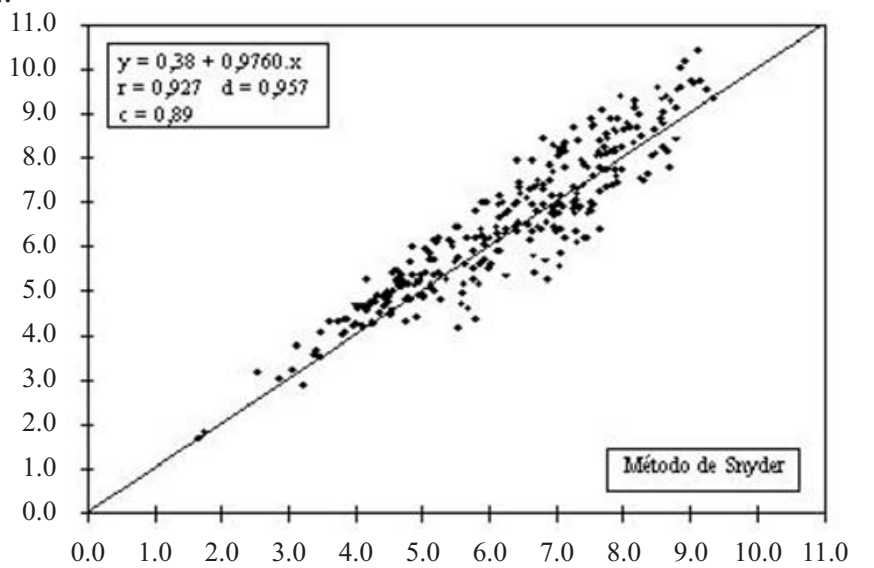

B.

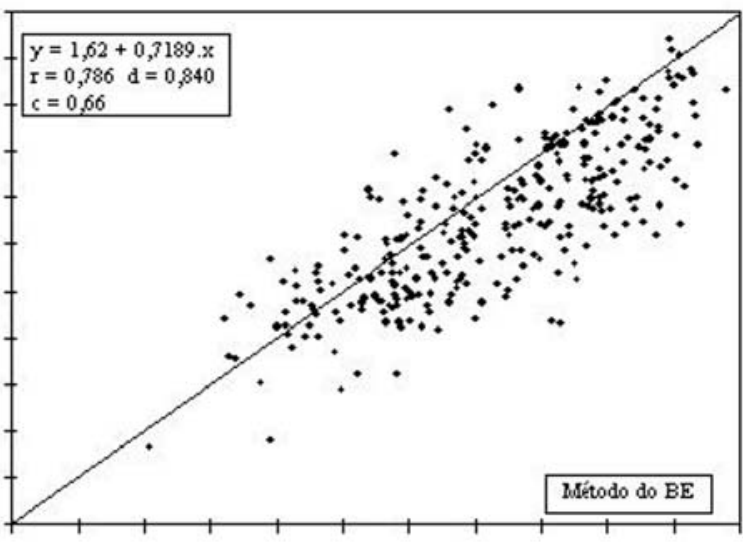

D.

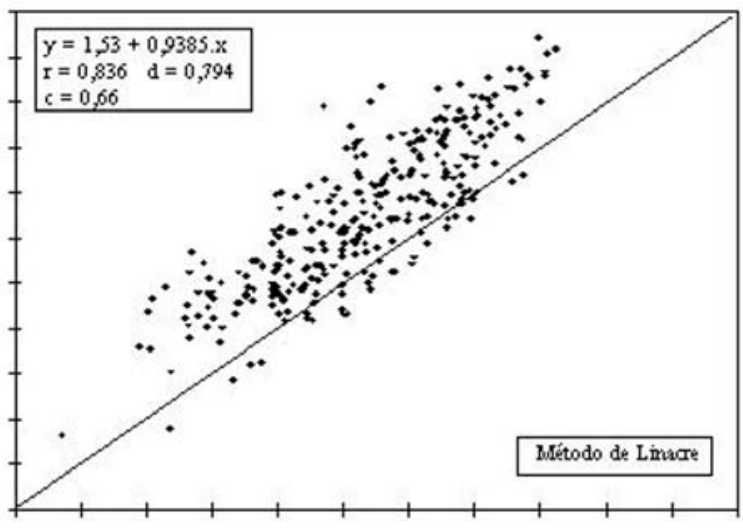

F.

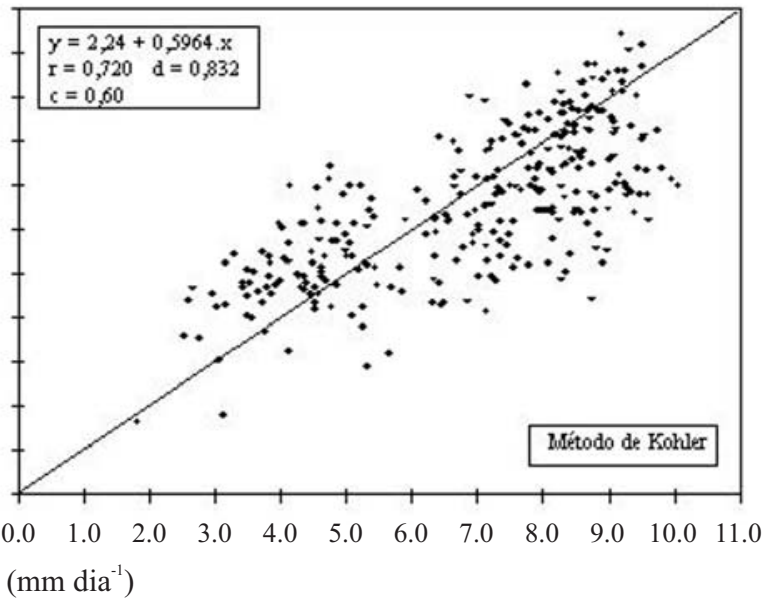

Figura 5. Dispersão para Patos, $\mathrm{PB}$, da evaporação estimada pelos métodos de (A) Penman (1948); (B) balanço de energia - BE; (C) Gangopadhyaya et al. (1966) e Hounam (1973) - G\&H; (D) Linacre (1993); (E) Snyder (1992) e (F) Kohler et al. (1955), em relação à evaporação observada

Boqueirão: 0,91; Patos: 0,89). Segundo Sartori (2000) os métodos que não consideram a UR prevêem estimativas de evaporação não exatas para o ar úmido. O tradicional método de Penman (1948) apresentou, em Patos, subestimação mais acentuada no período de maior incidência de ra- diação solar. Segundo Mahrt \& Ek (1984) a não inclusão da influência da estabilidade atmosférica sobre o transporte de vapor d’água no método de Penman e outros métodos, pode contribuir significativamente para a variação diurna da evaporação potencial. 


\section{CONCLUSÕES}

1. O método de Snyder (1992) sob todos os aspectos analisados foi o que apresentou o melhor desempenho nas duas regiões estudadas.

2. Os métodos do balanço de energia e Linacre (1993) tiveram melhor desempenho em Boqueirão que em Patos. O método de Linacre (1993) apresentou erro sistemático, logo pode ser ajustado e ter bom desempenho.

3. Os métodos de Penman (1948) e Kohler (1995) apresentaram os piores desempenhos em Patos com base nos indicadores estatísticos de Camargo \& Sentelhas (1997).

\section{LITERATURA CITADA}

Brutsaert, W.; Yeh, GT. Implications of a type of empirical evaporation formula for lakes and pans. Water Resources Research, Washington, v.6, n.4, p.1202-1208, 1970.

Camargo, A. P; Sentelhas, P. C. Avaliação do desempenho de diferentes métodos de estimativas da evapotranspiração potencial no Estado de São Paulo, Brasil. Revista Brasileira de Agrometeorologia, Santa Maria, v.5, n.1, p.89-97, 1997.

Chin, D. A.; Zhao, S. Evaluation of evaporation-pan networks. Journal of Irrigation and Drainage Engineering, ASCE, Jerusalem, v.121, n.5, p.338-346, 1995.

Doorenbos, J.; Pruitt, W. O. Guidelines for prediction of crop water requirements. FAO Irrigation and drainage Paper 24, 2.ed., Rome: FAO, 1977, 179p.

Finch, J. W.; Gash, J. H. C. Application of a simple finite difference model for estimating evaporation from open water. Journal of Hidrology, Amsterdam, v.255, p.253-259, 2002.

Gangopadhyaya, M.; Harbeck, G. E.; Nordenson, T. J.; Omar, M. H.; Uryvaev, V. A. Measurement and estimation of evaporation and evapotranspiration. Geneva: World Meteorological Organization, 1966. 121p. Technical Note n.83, WMO-No.201, TP 105.

Hounam, C. E. Comparison between pan and lake evaporation. Geneva; World Meteorological Organization. Rapporteur on Lake Evaporation, 1973. 52p. Technical Note No. 126.
Kohler, M. A.; Noredenson, T. J.; Fox, W. E. Evaporation from pans and lakes. U. S. Weather Bureau Research, 1955. 21p. Paper 38.

Linacre, E. T. Data-sparse estimation of lake evaporation using a simplified Penman equation. Agricultural and Forest Meteorology, Elsevier, Amsterdam, v.64, n.3-4, p.237-256, 1993.

Mahrt, L.; Ek, M. The influence of atmospheric stability on potential evaporation. Journal of Applied Meteorology, Boston, v.23, n.2, p.222-234, 1984.

Mero F.; Simon, E. Measurement and estimation of the evaporation from Lake Kinneret - 1973/74. Tahal Publication n. 01/75/ 29 (in Hebrew). p.291-303, 1975.

Oliveira, G. M. Estudo da evaporação nas microrregiões do Cariri e Sertão da Paraíba. Campina Grande: UFCG, 2003. 203p. Tese Doutorado

Penman, H. L. Natural evaporation from open water, bare soil and grass. Proceedings of the Royal Society. A - Series, London, v.193, p.120-145, 1948.

Sartori, E. A critical review on equations employed for the calculation of the evaporation rate from free water surfaces. Solar Energy, India, v.68, n.1, p.77-89, 2000.

Simon, E.; Mero F. A simplifield proceedure for the evaluation of the Lake Kinneret evaporation. Journal of Hidrology, Amsterdam, v.78, p.291-304, 1985.

Snyder, R. L. Equation for evaporation pan to evapotranspiratin conversions. Journal of Irrigation and Drainage Engineering, Jerusalem, v.118, n.6, p.977-980, 1992.

Willmott, C. J.; Ackleson, S. G.; Davis, R. E.; Feddema, J. J.; Klink, K. M.; Legates, D. R.; Rowe, C. M.; O’Donnell, J. Statistics for the evaluation and comparison of models. Journal of Geophysical Research, Ottawa, v.90, n.C5, p.89959005, 1985.

Winter, T. C.; Rosenberry, D. O.; Sturrock, A. M. Evaluation of 11 equations for determining evaporation for a small lake in the north central United States. Water Resources Research, Washington, v.31, n.4, p.983-994, 1995.

WMO - World Meteorological Organization. Guide to meteorological instruments and methods of observation. Geneva: WMO, 1996. cap.10, p.1-7. 